\title{
Does digital mammography suppose an advance in early diagnosis? Trends in performance indicators 6 years after digitalization
}

\author{
Maria Sala • Laia Domingo • Francesc Macià • \\ Mercè Comas • Andrea Burón • Xavier Castells
}

Received: 5 June 2014 /Revised: 19 August 2014 / Accepted: 3 September 2014 /Published online: 26 September 2014

(C) The Author(s) 2014. This article is published with open access at Springerlink.com

\begin{abstract}
Purpose To provide a complete evaluation of the long-term impact of full-field digital mammography (FFDM) on the improvement of early diagnosis in a population-based screening program.

Methods We included 82,961 screen-film mammograms (SFM) and 79,031 FFDM from women aged 50-69 screened biennially from 1995-2010 in Spain and followed-up to 2012. The first screening round of the program was excluded. Rates of cancer detection, interval cancer, tumoral characteristics and other quality indicators were compared between SFM and FFDM periods using the Chi-square test. Multivariate logistic regression models were fitted.

Results Detection of ductal carcinoma in situ (DCIS) significantly increased with FFDM (0.05\% vs $0.09 \% ; p=0.010)$, along with the proportion of small invasive cancers $(<20 \mathrm{~mm})$ $(69.37 \%$ vs $78.90 \% ; p=0.040)$. The false-positive rate decreased with FFDM $(4.79 \%$ vs $3.38 \% ; p<0.001)$ without differences in the cancer detection rate $(0.42 \%$ vs $0.43 \%$; $p=0.685)$ or in the interval cancer rate $(0.14 \%$ vs $0.14 \%$; $p=0.816$ ). Adjusted models showed a significant increase in the detection of DCIS in the FFDM periods.

Conclusion Digitalization has supposed an improvement in early diagnosis because DCIS and small invasive cancers increased without a change in detection rate. Moreover,
\end{abstract}

Maria Sala and Laia Domingo contributed equally to this work

M. Sala $(\bowtie) \cdot$ L. Domingo $\cdot$ F. Macià $\cdot$ M. Comas · A. Burón ·

X. Castells

Department of Epidemiology and Evaluation, IMIM (Hospital del

Mar Medical Research Institute), Pg. Marítim 25-29,

08003 Barcelona, Spain

e-mail: MSalaSerra@parcdesalutmar.cat

M. Sala $\cdot$ L. Domingo $\cdot$ F. Macià $\cdot$ M. Comas $\cdot$ A. Burón

X. Castells

Research Network on Health Services in Chronic Diseases

(REDISSEC), Pg. Marítim 25-29, 08003 Barcelona, Spain false-positive reduction without an increase in the interval cancer rate was confirmed.

Key Points

- Cancer detection did not increase after 6 years of digital mammography

- Ductal carcinoma in situ rates remained higher throughout the digital period

- The proportion of small invasive cancers was higher with digital mammography

- We observed an improvement in early diagnosis with digital mammography

- False-positive rates remained lower throughout the digital period without interval cancer increase

Keywords Mass screening · Mammography · Diagnostic imaging $\cdot$ Breast neoplasms $\cdot$ Carcinoma in situ
Abbreviations
DCIS Ductal carcinoma in situ
FFDM Full-field digital mammography
PPV Positive predictive value
SFM Screen film mammography

\section{Introduction}

The main goal of mammography screening is to reduce mortality and morbidity from breast cancer through early detection. The diagnostic accuracy of full-field digital mammography (FFDM) is clearly at least as good as that of screen-film mammography (SFM) [1]. However, there are some controversies on the specific effect on different performance indicators, including the detection of ductal carcinoma in situ (DCIS). Recent studies have shown that the detection rate is higher with FFDM than with SFM, partly owing to greater 
detection of DCIS [2, 3]. Some studies providing data on the characteristics of tumours detected by FFDM have described a trend to identify less advanced invasive cancers [2, 4]. These results point to two possible situations: an improvement in early diagnosis or, on the contrary, a worrying increase in overdiagnosis. The effect of the switch to FFDM on recall rate and positive predictive value (PPV) is unclear, with some studies showing a higher recall rate and lower PPV [2, 5], while others report a lower recall rate $[6,7]$ and similar or higher PPV [8, 9]. The effect on interval cancer has been studied less, but several reports have found no effect of FFDM on interval cancer rate $[6,10,11]$.

However, to our knowledge, no studies have presented data on all these quality indicators together (cancer rates, including interval cancer, sensitivity, specificity, PPV and tumour characteristics). Moreover, one of the main limitations of studies comparing the two technologies is the short time period since the introduction of FFDM. Most of the studies provide data only from the first one or two screening rounds performed with FFDM [2, 5, 7-9, 12], which may have been influenced by the transition from one technology to the other, as well as by the learning curve. Therefore, the evolution of quality indicators in subsequent digital screening rounds has scarcely been evaluated. Evaluation of the long-term effect of FFDM on the screening performance indicators within a cohort perspective is required for a complete evaluation of screening.

The aim of this study was to analyse trends in the cancer detection rate (invasive cancers and DCIS), tumoral characteristics, interval cancer rate and the sensitivity, specificity and PPV of FFDM over a 16-year period with 6 years of complete digitalization.

\section{Patients and methods}

Setting and study population

A retrospective cohort study was performed in women participating in a population-based breast cancer screening program in an area of 300,000 inhabitants in the city of Barcelona, Spain. Women aged 50-69 years were invited by personal letter to undergo mammography with a 2-year interval between screening rounds. We included data from 61,859 participating women from 1 November 1995 to 31 December 2010, and followed up until 31 December 2012. These women were screened in two radiology units and underwent a total of 182,002 screens.

The two radiology units began screening activities with SFM (SSH 140 A; Toshiba and Bennett, Trex Medical, Copiague, NY. Film: Mamoray-HT, AGFA, Greenville, SC) in 1995 and 1999, respectively, and shifted to FFDM (DM 1000 Agfa; Lorad, Danbury, Conn) in 2007 and 2004, respectively. Mediolateral oblique and craniocaudal views were available for each breast. All mammograms were read by two radiologists using the BI-RADS classification [13], and when double reading led to different assessments, a third radiologist served as a tiebreaker. Prior screening mammograms were always available in the original format during reading in successive screenings.

The program was based on the European Guidelines for Quality Assurance in Mammographic Screening [14] and its results met the Europe Against Cancer standards. The study was approved by the Ethics Committee of Parc de Salut Mar. Informed consent was not required.

\section{Screening procedures}

The screening program keeps mammogram registers with data from participants and the final outcome of screening. Two results of a screening test are possible: normal findings (for which screening mammography at 2 years is recommended) and abnormal findings, which require further assessments to confirm or exclude malignancy. When, after further assessments, a tumour is found (DCIS or invasive cancer), the result is considered a true positive. Otherwise, the result is considered a false positive.

Further assessments can include noninvasive procedures (additional mammography, ultrasound, magnetic resonance imaging) and/or invasive procedures (fine-needle aspiration, core-needle biopsy, open biopsy). Once a malignant tumour is histologically confirmed, the woman is sent to the referral hospital for treatment and follow-up. These women are not invited to further screening.

After a negative result of a screening episode, with or without further assessments, and before the next screening invitation, a woman may be diagnosed with interval breast cancer [14]. Interval cancers were identified by merging data from the register of the screening program with data from the hospital-based cancer registry and telephone contact with women who underwent mammography in the last scheduled screening but who did not attend the following screening invitation. This procedure covered $98 \%$ of women lost to follow-up from the program [15]. In our study, we extended the definition of interval cancer until the 30th month, because each screening round can last up to 6 months. All data sources kept information on the date of diagnosis, which allowed us to ensure that all interval cancers fitted the case definition.

This study included 161,992 screening mammograms performed from the second screening round onwards $(82,961$ SFM and 79,031 FFDM). In all, 684 tumours were detected in screening ( 345 with SFM and 339 with FFDM), and 226 tumours were diagnosed as interval cancers (114 with SFM and 112 with FFDM) (Fig. 1). 
Fig. 1 Flow chart of the study. Parc de Salut Mar breast cancer screening program, Barcelona, 1995-2010

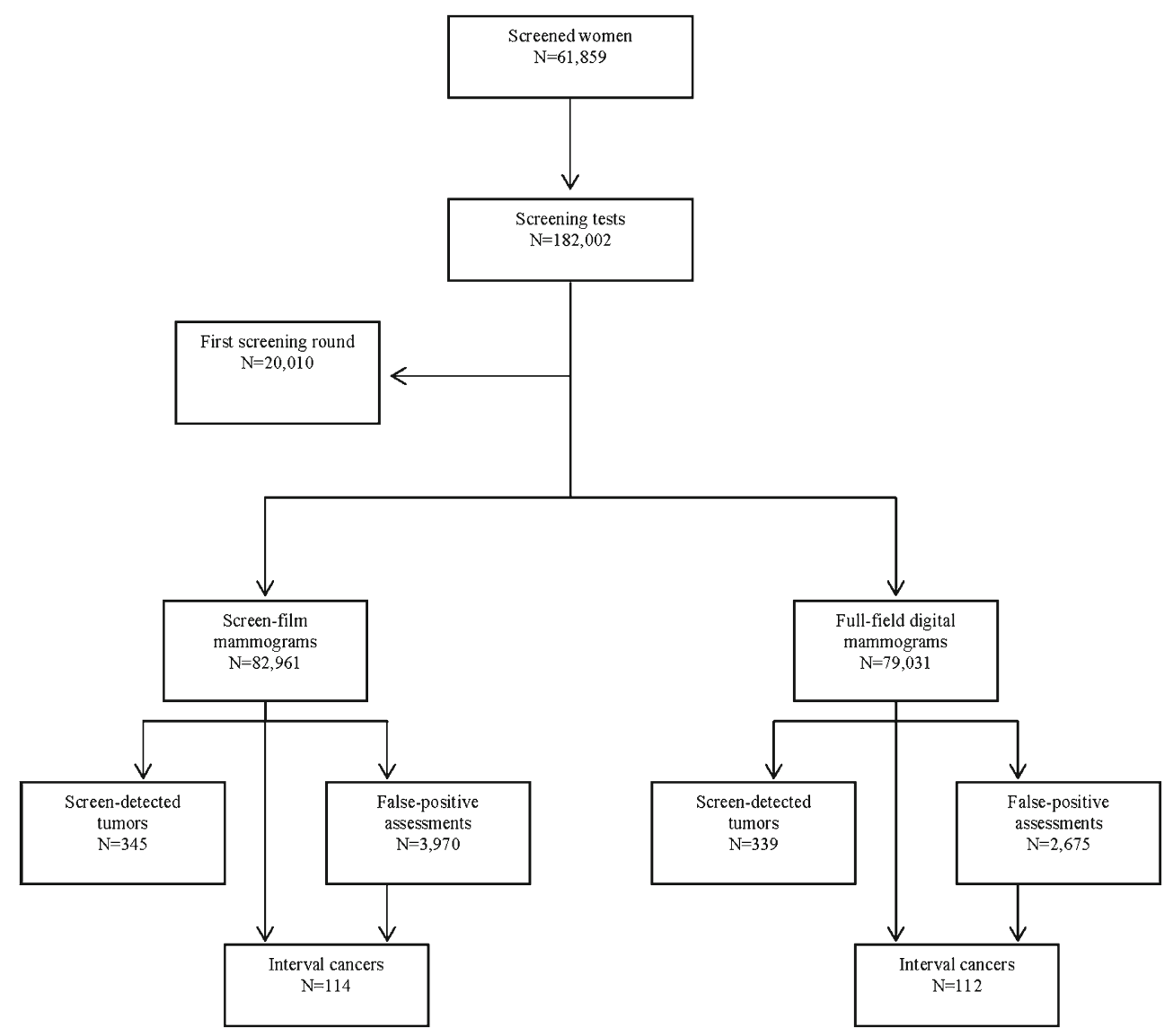

\section{Study period}

For the purpose of this study, the first screening round after the implementation of the screening program in both radiology units (RU1 and RU2) (prevalent round, all performed with SFM) was excluded from the analyses. We excluded the first round of the screening program because of its particular characteristics, such as the prevalence peak of cancer detection and the higher proportion of larger tumours, which could bias our results. Thus, we excluded all screening mammograms performed from November 1995 to December 1997 for RU1 and from January 1999 to December 2000 in RU2 $(n=20,010)$. Overall, 9 years were covered by SFM (from January 1998 to March 2007-9 years - in RU1, and from January 2001 to September 2004-3 years - in RU2). Similarly, the digital period covered 6 years (from March 2007 to December 2010 - 3 years - in RU1, and from September 2004 to December 2010 in RU2 - 6 years). Both radiology units cover two closed neighbourhoods in Barcelona and use the same screening protocol. The mammograms were read by the same radiologists.

Study variables

Information from screening (initial or successive screening, date of screening mammogram, further assessments, the use of SFM or FFDM, and the final outcome of screening) was obtained from the screening program database.

Age at diagnosis was obtained from the date of birth and date of the screening mammogram.

Tumour-related information (invasiveness, pathological tumour-node-metastasis [TNM] status and histological grade) was drawn from the hospital-based cancer registry.

\section{Statistical analyses}

Cancer detection rates (overall, invasive and DCIS) were computed as the number of cancers detected per 100 screening tests performed with SFM and FFDM. To calculate the interval cancer rate, women with cancer diagnosed at screening were excluded from the denominator, because they were not "at risk" of an interval cancer. Recall rate was defined as the percentage of screened women requiring at least one further assessment after a positive mammogram. False-positive rates were defined as the number of further assessments (including noninvasive and/or invasive procedures) with no cancer diagnosis divided by the number of screening tests, performed with SFM and FFDM.

To calculate the accuracy measures, we used the definitions from the European Guidelines for Quality Assurance in Mammographic Screening [14]. Sensitivity was computed as the 
proportion of cancers identified at screening (screen-detected cancers) divided by the sum of screen-detected cancers and interval cancers. Specificity was the proportion of truly negative screening examinations relative to true negatives plus false positives. The PPV of the screening test was computed as the proportion of screen-detected cancers divided by the sum of false positives and screen-detected cancers. Proportions were compared by the two-sided Chi-square test.

We computed a time/technique variable, which allowed us to exclude possible confounding due to time trends and to analyse information from the two radiology units together, because they covered different periods using SFM and FFDM $[4,7]$. We divided the screening history in each radiology unit into four equal time intervals (quartiles) both for the SFM period and FFDM period. Therefore, each time interval for SFM and DM had a similar number of screening tests.

Logistic regression analyses were performed to assess the odds of cancer detection and interval cancer in the SFM and FFDM periods, adjusting for radiology unit, age and whether the diagnosis was established at the initial or successive screenings. The logistic regression models were replicated to assess the odds of invasive cancer and DCIS detection. For the former, we censored DCIS diagnostics and for the latter, we censored invasive diagnostics. Repeated measures in the logistic models were considered to be independent observations, because cancer detection always depends on the absence of a previous diagnosis. The outputs of the logistic regression models were plotted, showing the adjusted odds ratios (OR) and the $95 \%$ confidence intervals $(95 \% \mathrm{CI})$.

We conducted a sensitivity analysis by including versus excluding the 3-month learning period after the switch to digital technology. We also compared the results by including the program's first screening round.

All $p$ values were based on two-sided tests and were considered statistically significant if less than 0.05 . Statistical analyses were performed using the SPSS (version 12.0).

\section{Results}

The overall cancer detection rate showed no statistically significant differences between the SFM and FFDM periods ( 0.42 vs $0.43 \% ; p=0.685$, Table 1 ). However, the detection rate of DCIS rose during the FFDM period $(0.05 \%$ vs $0.09 \%$; $p=0.010)$, only in the initial screenings $(0.06 \%$ vs $0.12 \%$; $p=0.031$ ). The rate of interval cancers showed no statistically significant differences between the two study periods $(0.14 \%$ vs. $0.14 \%$, respectively; $p=0.816$ ), whereas the rate of false positives dramatically decreased during the FFDM period, from $4.79 \%$ to $3.38 \%(p<0.001)$.

Table 2 shows tumour-related characteristics according to whether cancers were detected by SFM or FFDM. Tumours detected by FFDM tended to be diagnosed at earlier stages, the proportion of DCIS being $20.30 \%$ in FFDM and $13.14 \%$ in SFM ( $p=0.092)$. When invasive cancers only were considered, the proportion of cancers smaller than $20 \mathrm{~mm}$ was significantly higher during the FFDM period than during the SFM period ( $78.90 \%$ vs. $69.37 \%$, respectively; $p=0.040$ ). In DCIS, the percentage of high-grade tumours was higher in those detected with FFDM than with SFM, although this difference was not statistically significant $(60.34 \%$ vs. $51.22 \%$, respectively; $p=0.581$ ).

Figure 2 shows the distribution of tumour size of invasive screen-detected cancers by study period. During the digital periods, the percentages of invasive cancers smaller than $20 \mathrm{~mm}$ tended to increase $(73.1 \%$ in the 4th SFM period, and 80.3 in the 4th FFDM period; $p=0.095$ ), whereas the proportion of T2 (tumours from 20 to $50 \mathrm{~mm}$ ) became smaller in comparison with the SFM periods $(16.4 \%$ in the 4 th SFM period and $11.5 \%$ in the 4th FFDM period; $p=0.095$ ).

Table 3 shows the rates of screen-detected cancers, interval cancers, false positives, recall rate and accuracy measures during the study period. The rate of screen-detected cancers remained fairly stable across the study periods, with no particular trend. The interval cancer rate was $0.13 \%$ in the first SFM and increased to $0.21 \%$ in the first FFDM period. Thereafter, it gradually declined, reaching the lowest values in the last FFDM period $(0.11 \%)$. The false-positive rate and the recall rate showed a decreasing trend over time, especially from the beginning of the digital period. Sensitivity, specificity and PPV increased during the digital period.

Rates of screen-detected cancers, invasive cancer and DCIS over time are shown in Fig. 3. Invasive cancer rates slightly decreased in the last FFDM periods, while those of DCIS increased over the same periods.

Figure 4 plots the adjusted OR for cancer detection at screening (overall, invasive and DCIS) and for interval cancers. The introduction of FFDM did not increase cancer detection at screening, although the risk increased in the 2 nd and 4th FFDM periods, without reaching statistical significance [2nd DM period, OR=1.19 (95\% CI 0.88-1.62); 4th FFDM period, $\mathrm{OR}=1.14(95 \% \mathrm{CI} 0.84-1.55)]$. The highest detection of interval cancer was found in the first FFDM period $[\mathrm{OR}=1.69(95 \% \mathrm{CI} 1.03-2.77)]$ but the trend then dramatically decreased over the subsequent FFDM periods. In the FFDM periods, the risk of DCIS detection increased significantly from an $\mathrm{OR}=1.58$ (95\% CI 0.65-3.80) in the first FFDM period to an $\mathrm{OR}=2.68(95 \%$ CI 1.19-6.00) in the fourth DM period. Nevertheless, detection of invasive cancers did not show a clearly declining pattern in the adjusted model.

The results of the sensitivity analysis revealed that inclusion versus exclusion of the 3-month learning period after the switch to digital technology did not significantly affect any of the screening indicators presented. Equally, the inclusion of 
Table 1 Screening performance indicators for screen-film mammography and digital mammography
${ }^{\text {a }}$ Chi-square test

${ }^{\mathrm{b}}$ The total number of screened women is not the sum of women screened with screen-film and full-field digital mammography, because some women were screened during both periods $(n=23,126)$

${ }^{\mathrm{c}}$ The interval cancer rate was calculated as the number of interval cancers over the number of screened women minus women with screen-detected cancer in the same period

${ }^{\mathrm{d}}$ Noninvasive and invasive procedures

\begin{tabular}{|c|c|c|c|c|c|}
\hline & \multicolumn{2}{|c|}{ Screen-film mammography } & \multicolumn{2}{|c|}{ Digital mammography } & \multirow[t]{2}{*}{$p$ value $^{\mathrm{a}}$} \\
\hline & $n$ & $\%$ & $n$ & $\%$ & \\
\hline Screened women ${ }^{\mathrm{b}}$ & 39,182 & & 43,406 & & \\
\hline Screening mammograms & 82,961 & & 79,031 & & \\
\hline Initial screening & 21,187 & & 14,532 & & \\
\hline Successive screening & 61,774 & & 64,499 & & \\
\hline Cancer detection rate & 345 & 0.42 & 339 & 0.43 & 0.685 \\
\hline Initial screening & 82 & 0.39 & 80 & 0.55 & 0.024 \\
\hline Successive screening & 263 & 0.43 & 259 & 0.40 & 0.503 \\
\hline Invasive carcinomas detection rate & 295 & 0.36 & 264 & 0.33 & 0.462 \\
\hline Initial screening & 66 & 0.31 & 61 & 0.42 & 0.091 \\
\hline Successive screening & 229 & 0.37 & 203 & 0.31 & 0.089 \\
\hline In situ carcinoma detection rate & 45 & 0.05 & 70 & 0.09 & 0.010 \\
\hline Initial screening & 12 & 0.06 & 18 & 0.12 & 0.031 \\
\hline Successive screening & 33 & 0.05 & 52 & 0.08 & 0.063 \\
\hline Interval cancer rate ${ }^{c}$ & 114 & 0.14 & 112 & 0.14 & 0.816 \\
\hline Initial screening & 39 & 0.18 & 32 & 0.22 & 0.447 \\
\hline Successive screening & 75 & 0.12 & 80 & 0.12 & 0.895 \\
\hline Recall rate & 4,628 & 5.57 & 3,316 & 4.20 & $<0.001$ \\
\hline Initial screening & 2,331 & 11.00 & 1,705 & 11.73 & 0.032 \\
\hline Successive screening & 2,297 & 3.72 & 1,611 & 2.50 & $<0.001$ \\
\hline False-positive for any procedures ${ }^{\mathrm{d}}$ & 3,970 & 4.79 & 2,675 & 3.38 & $<0.001$ \\
\hline Initial screening & 1,872 & 8.84 & 1,165 & 8.02 & 0.006 \\
\hline Successive screening & 2,098 & 3.40 & 1,510 & 2.34 & $<0.001$ \\
\hline Sensitivity & & 75.16 & & 75.17 & 0.960 \\
\hline Initial screening & & 67.77 & & 71.43 & 0.877 \\
\hline Successive screening & & 77.81 & & 76.40 & 0.921 \\
\hline Specificity & & 95.19 & & 96.60 & 0.040 \\
\hline Initial screening & & 91.11 & & 91.92 & 0.578 \\
\hline Successive screening & & 96.59 & & 97.65 & 0.175 \\
\hline Positive predictive value & & 8.00 & & 11.25 & $<0.001$ \\
\hline Initial screening & & 4.20 & & 6.43 & 0.010 \\
\hline Successive screening & & 11.14 & & 14.64 & 0.004 \\
\hline
\end{tabular}

the first screening round did not modify the direction of our findings.

\section{Discussion}

No differences were observed in overall cancer detection rates between the SFM and FFDM periods. However, DCIS rates increased with digital mammography, and remained higher throughout the digital periods, while the invasive carcinoma detection rate was somewhat lower in the FFDM periods. Information on tumour characteristics pointed to a stage shift, especially when we analysed the size of invasive tumours. The proportion of smaller invasive tumours tended to increase during the FFDM periods. The proportion of high-grade DCIS was also higher, but not statistically significant, in the FFDM period. False-positive rates remained lower in all the digital periods with no increase in the interval cancer rate. No differences were observed in sensitivity between the two periods, but specificity and especially PPV increased throughout the FFDM periods. The adjusted screen-detected cancer (overall and invasive) and interval cancer rates showed no statistically significant differences throughout the study period, but detection of DCIS increased in the FFDM period.

Most studies comparing the two technologies have detected an increase in DCIS detection rates with FFDM [2, 4, 6, 7, $9,12]$. In agreement with our results, the increase has been observed especially in initial screenings [4, 7] but also in successive ones $[4,7,9]$. The significance of this increase 
Table 2 Tumour-related characteristics of cancers detected by screen-film mammography and full-field digital mammography
${ }^{\mathrm{a}}$ The number of in situ and invasive carcinomas is not the sum of overall cancers, because data are missing on some tumours (5 tumours in the SFM period and 5 tumours in the FFDM period)

${ }^{\mathrm{b}}$ Tumours with missing information were excluded from the calculation of percentages

\begin{tabular}{|c|c|c|c|c|c|}
\hline & \multicolumn{2}{|c|}{ Screen-film mammography } & \multicolumn{2}{|c|}{ Digital mammography } & \multirow[t]{2}{*}{$p$ value } \\
\hline & $n=340^{\mathrm{a}}$ & $\%$ & $n=334^{\mathrm{a}}$ & $\%$ & \\
\hline \multicolumn{6}{|l|}{ TNM stage } \\
\hline Stage 0 (in situ) & 41 & 13.14 & 63 & 20.32 & \\
\hline Stage I & 156 & 50.00 & 157 & 50.65 & \\
\hline Stage II & 91 & 29.17 & 69 & 22.26 & \\
\hline Stage III & 21 & 6.73 & 19 & 6.13 & \\
\hline Stage IV & 3 & 0.96 & 2 & 0.65 & 0.092 \\
\hline Unknown $^{\mathrm{b}}$ & 28 & & 24 & & \\
\hline \multicolumn{6}{|c|}{ TNM stage (invasive only) } \\
\hline Stage I & 156 & 57.56 & 157 & 63.56 & \\
\hline Stage II & 91 & 33.58 & 69 & 27.90 & \\
\hline Stage III & 21 & 7.74 & 19 & 7.70 & \\
\hline Stage IV & 3 & 1.11 & 2 & 0.80 & 0.528 \\
\hline Unknown $^{\mathrm{b}}$ & 28 & & 24 & & \\
\hline \multicolumn{6}{|c|}{ Tumour size (invasive only) } \\
\hline $\mathrm{T} 1(<20 \mathrm{~mm})$ & 188 & 69.37 & 194 & 78.90 & \\
\hline $\mathrm{T} 2(20$ to $50 \mathrm{~mm})$ & 58 & 21.40 & 32 & 13.00 & \\
\hline $\mathrm{T} 3(>50 \mathrm{~mm})$ & 16 & 5.90 & 16 & 6.50 & \\
\hline $\mathrm{T} 4$ & 9 & 3.32 & 4 & 1.60 & 0.040 \\
\hline $\mathrm{Tx}^{\mathrm{b}}$ & 28 & & 24 & & \\
\hline \multicolumn{6}{|c|}{ Lymph node involvement (invasive only) } \\
\hline No & 197 & 72.70 & 181 & 73.28 & \\
\hline $\mathrm{N} 1-\mathrm{N} 3$ & 74 & 27.30 & 66 & 26.72 & 0.959 \\
\hline $\mathrm{Nx}^{\mathrm{b}}$ & 28 & & 24 & & \\
\hline \multicolumn{6}{|c|}{ Metastasis (invasive only) } \\
\hline M0 & 268 & 98.90 & 245 & 99.59 & \\
\hline M1 & 3 & 1.10 & 2 & 0.81 & 0.730 \\
\hline $\mathrm{Mx}^{\mathrm{b}}$ & 28 & & 24 & & \\
\hline \multicolumn{6}{|l|}{ Tumour grade } \\
\hline \multicolumn{6}{|c|}{ Ductal carcinoma in situ } \\
\hline I & 8 & 19.51 & 11 & 18.97 & \\
\hline II & 12 & 29.27 & 12 & 20.69 & \\
\hline III & 21 & 51.22 & 35 & 60.34 & 0.581 \\
\hline Unknown $^{\mathrm{b}}$ & 4 & & 12 & & \\
\hline \multicolumn{6}{|l|}{ Invasive cancers } \\
\hline I & 86 & 36.91 & 95 & 43.78 & \\
\hline II & 102 & 43.78 & 68 & 31.34 & \\
\hline III & 45 & 19.31 & 54 & 24.88 & 0.023 \\
\hline Unknown $^{\mathrm{b}}$ & 62 & & 47 & & \\
\hline
\end{tabular}

on DCIS has been little studied and it is not known whether it is due to earlier diagnosis [4, 6] or to overdiagnosis [2].

An earlier diagnosis should be followed by a decrease of invasive cancers or at least by detection of less advanced invasive cancers. Our results pointed to an earlier diagnosis, showing not only significant differences in TNM stages, with a higher proportion of DCIS in the FFDM period, but also a higher proportion of smaller, invasive tumours along the FFDM periods, which has also been reported by other authors [6]. In line with our findings, recent work by Drukker et al. reported a higher detection of biologically high-risk cancers with FFDM [16]. Moreover, as previously reported [17], among DCIS we found a higher proportion of high-grade tumours in the FFDM period, although this difference was 


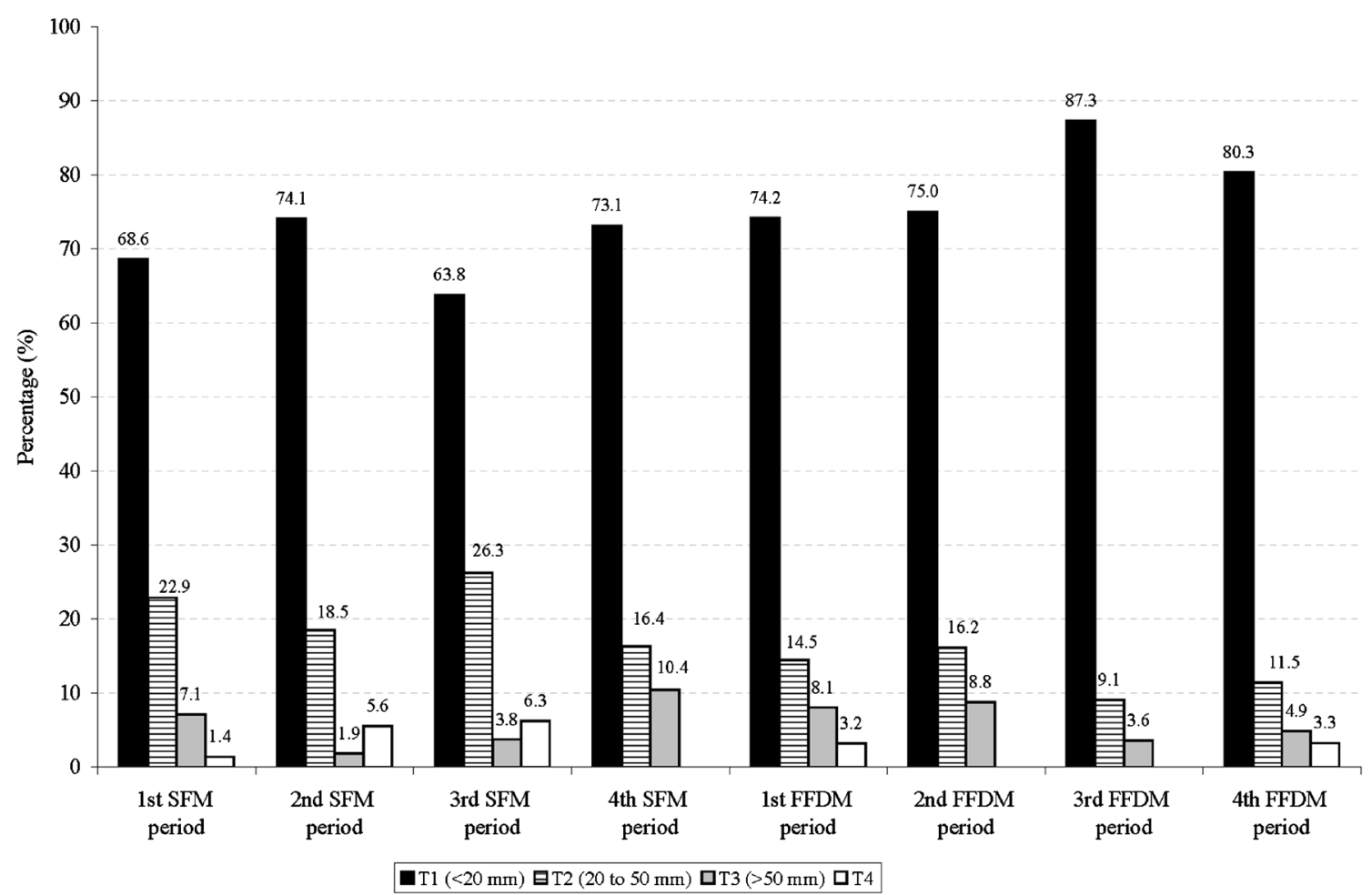

Fig. 2 Distribution of tumour size of invasive screen-detected cancers by study periods

not statistically significant. Because low-grade DCIS may have a lower potential to become invasive $[18,19]$, the similar proportions observed in the two periods do not support the hypothesis of overdiagnosis. However, larger series are necessary to confirm this trend.

After the shift to FFDM, numerous studies in Spain $[7,20]$ and elsewhere $[21,22]$ observed a reduction in the recall rate, which led to suspicions of an increase in interval cancers and false negatives. The current work, in agreement with previous studies $[6,11]$, confirms that the reduction in recall rate was not due to an increase in interval cancer during the FFDM period. Although we have no information on interval cancer subtypes, previously published data refute the hypothesis that the introduction of FFDM has increased the number of falsenegative cancers $[10,11]$. However, an increasing trend in the detection of interval cancers was observed in the SFM periods, with the highest interval cancer rate in the first FFDM period and a clear subsequent decrease. These increases could be partly attributed to an improvement in the mechanisms to detect interval cancers introduced in the screening programs during their implementation (active follow-up of women, merging data from the screening program registers with the regional Minimum Basic Data Set—based on hospital discharges with information on the principal diagnosis - and

Table 3 Rates of screen-detected cancers, interval cancers, false positives and accuracy measures by study period

\begin{tabular}{|c|c|c|c|c|c|c|c|c|c|c|c|c|}
\hline & \multirow[t]{2}{*}{$\begin{array}{l}\text { Screening } \\
\text { mammograms }\end{array}$} & \multicolumn{2}{|c|}{$\begin{array}{l}\text { Screen-detected } \\
\text { cancers }\end{array}$} & \multicolumn{2}{|c|}{ Interval cancers } & \multicolumn{2}{|c|}{ Recall rate } & \multicolumn{2}{|c|}{ False-positives } & \multirow[t]{2}{*}{ Sensitivity } & \multirow[t]{2}{*}{ Specificity } & \multirow[t]{2}{*}{$\begin{array}{l}\text { Positive predictive } \\
\text { value }\end{array}$} \\
\hline & & $n$ & $\%$ & $n$ & $\%$ & $n$ & $\%$ & $n$ & $\%$ & & & \\
\hline 1st SFM period & 20,760 & 86 & 0.41 & 27 & 0.13 & 1,225 & 5.90 & 1,026 & 4.94 & 76.11 & 95.03 & 7.73 \\
\hline 2nd SFM period & 20,757 & 72 & 0.35 & 21 & 0.10 & 1,132 & 5.45 & 970 & 4.67 & 77.42 & 95.31 & 6.91 \\
\hline 3rd SFM period & 20,729 & 102 & 0.49 & 39 & 0.19 & 1,186 & 5.72 & 1,039 & 5.01 & 72.34 & 94.95 & 8.94 \\
\hline 4th SFM period & 20,714 & 85 & 0.41 & 27 & 0.13 & 1,084 & 5.23 & 934 & 4.51 & 75.89 & 95.47 & 8.34 \\
\hline 1st FFDM period & 19,733 & 81 & 0.41 & 42 & 0.21 & 815 & 4.13 & 678 & 3.44 & 65.85 & 96.54 & 10.67 \\
\hline 2nd FFDM period & 19,760 & 91 & 0.46 & 27 & 0.14 & 960 & 4.86 & 800 & 4.05 & 77.12 & 95.93 & 10.21 \\
\hline 3rd FFDM period & 19,780 & 80 & 0.40 & 22 & 0.11 & 750 & 3.79 & 594 & 3.00 & 78.43 & 96.98 & 11.87 \\
\hline 4th FFDM period & 19,701 & 87 & 0.44 & 21 & 0.11 & 788 & 4.00 & 600 & 3.05 & 80.56 & 96.94 & 12.66 \\
\hline
\end{tabular}


Fig. 3 Rates of overall screendetected cancer, invasive cancer and ductal carcinoma in situ (DCIS) over the study period

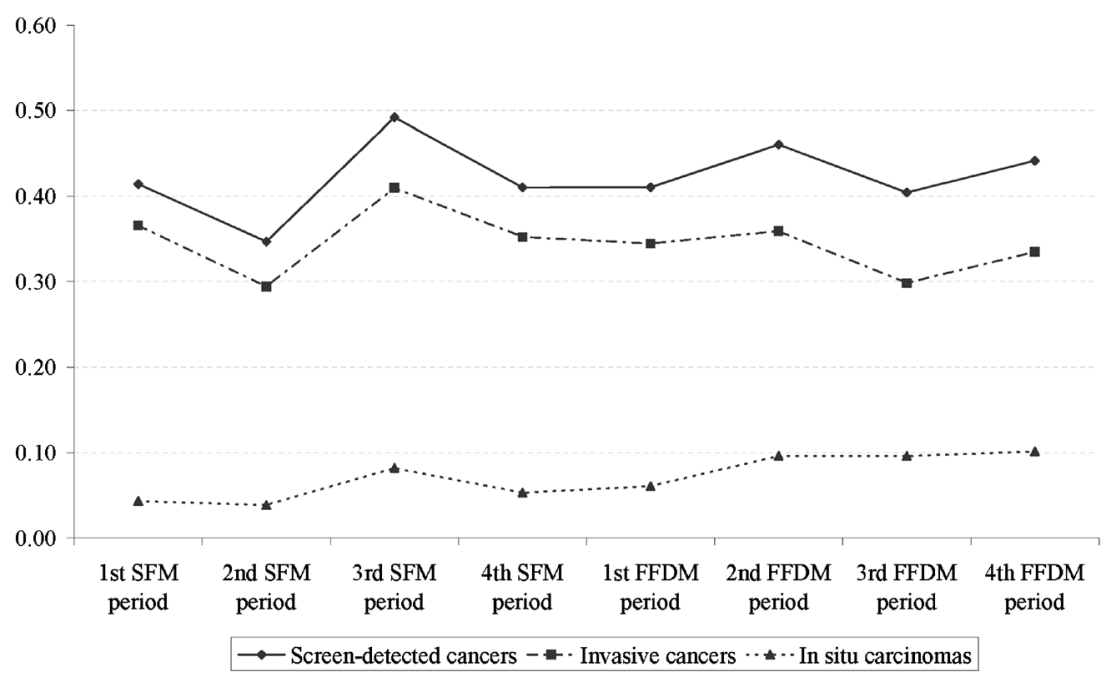

hospital-based cancer registries). However, after the shift to FFDM, no further changes were made to those mechanisms.

Some studies have described a reduction in PPV after the introduction of FFDM [2, 5], whereas others [4, 6-8, 12], including ours, have shown an increase, especially in successive screenings. The implementation of digital technology in our population-based screening program reduced the number of adverse effects related to false-positive results and costs [23], with similar cancer detection and interval cancer rates.
Because cancer detection rates are affected by other factors, a logistic model was used, adjusting by radiology unit, initial and successive screening, and age. Overall, the adjusted OR of detection of cancer or interval cancer remained unchanged throughout the period. However, the odds of DCIS detection were higher in the FFDM periods than in the SFM periods. To date, no other studies have adjusted the risk by time trends; a recent Norwegian study found that the risk of DCIS detection was increased during the whole FFDM period, but no
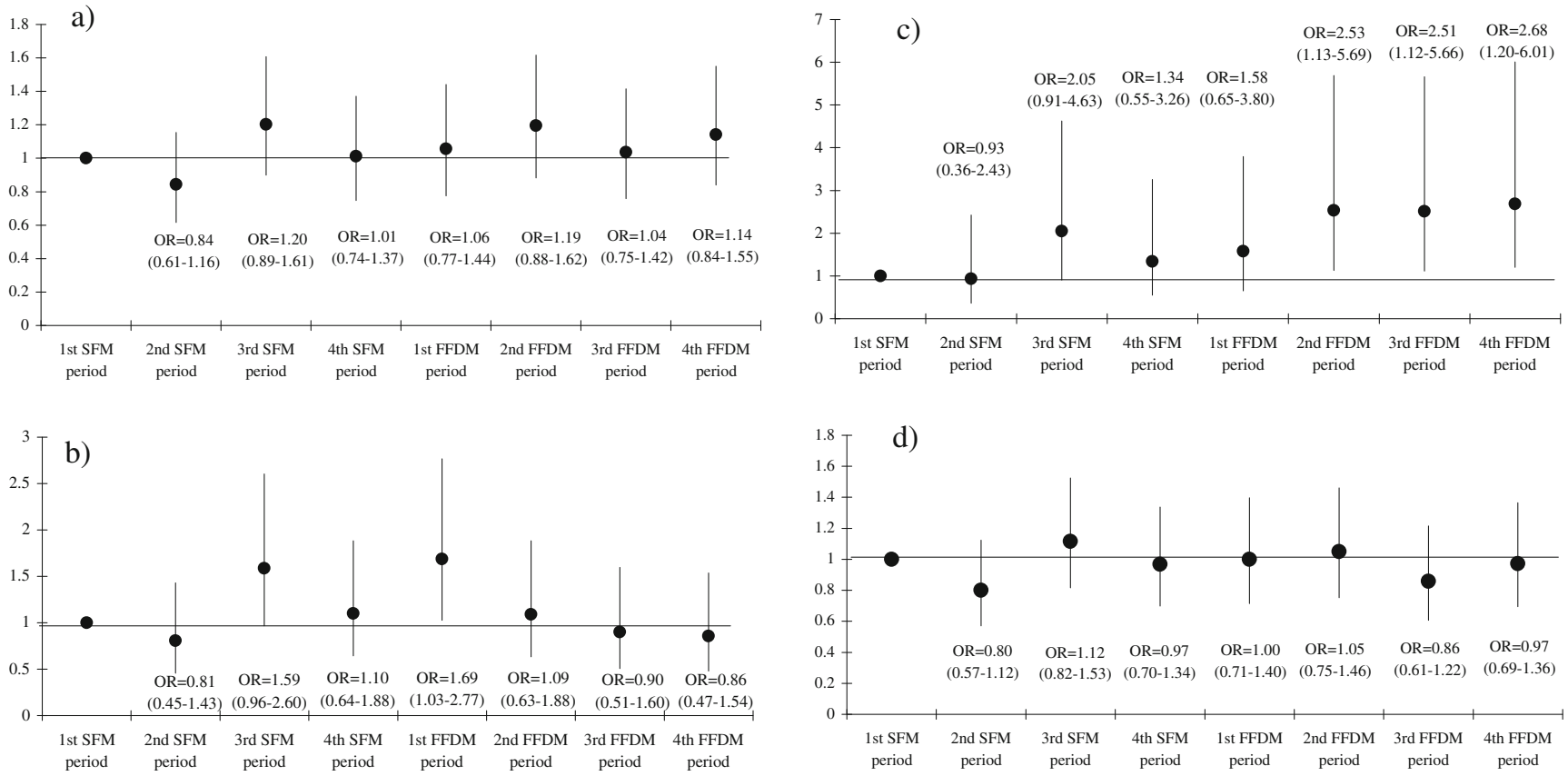

Fig. 4 Logistic regression models for the detection of screen-detected cancer, interval cancer, ductal carcinoma in situ (DCIS) and invasive cancer. The models are adjusted by radiology unit, age (continuous) and whether the diagnosis was established at the initial or successive screenings. a Screen-

detected cancers, $\mathbf{b}$ interval cancers, $\mathbf{c}$ ductal carcinoma in situ (DCIS), $\mathbf{d}$ invasive cancers. The reference category is the first screen-film mammography period. The black points and the vertical lines represent the odds ratio (OR) and the corresponding $95 \%$ confidence intervals, respectively 
increased risk was found for screen-detected cancers overall, invasive cancers or interval cancers [6].

This study has some limitations. Although the study period is one of the longest ever analysed, the number of DCIS does not allow exploration of trends in tumoral grade during the digital period. We had no information on interval cancer subtypes, and therefore we could not assess the behaviour of false negatives before and after the shift to digital technology.

The main strength of the current work is the long study period, with more than 6 years of complete digitalization. This allowed us to study trends over time, beyond the first digital period after the transition. Because of the wide variability observed in the performance indicators of screening programs, both within and between countries, cross-sectional observations cannot reflect the real impact of one intervention. Differences observed in a given screening participation (screening round) may be compensated for in subsequent screening participations. After confirming that the direction of our findings was not modified, we excluded the first screening round of both the radiology units, both of which used SFM, which reduced the sample size in the analogical period but ensured that the indicators in the SFM period were not confounded by the prevalence peak after the implementation of screening, or by the larger tumour sizes found in the first screening round [24]. We included the first 3 months after the switch to digital mammography because it did not modify the outcomes.

In conclusion, this study supports the idea that the increase in DCIS detection observed with the introduction of FFDM is partly due to an improvement in early diagnosis and confirms the reduction in the false-positive rate with no increase in the interval cancer rate. In view of the current results, which are supported by those from previous studies performed in different countries, the use of digital technology should not be seen as a threat that increases the negative effects of screening through overdiagnosis. Future recommendations on screening performance and quality standards should be updated with information from screening settings using this technology.

\footnotetext{
Acknowledgments The authors thank Cristina Hernández for her contribution in the data collection from the screening program and Isabel Torá-Rocamora for her assistance in analysis and data management. We also thank the Hospital del Mar Cancer Registry for providing tumourrelated data. The scientific guarantor of this publication is Maria Sala. The authors of this manuscript declare no relationships with any companies whose products or services may be related to the subject matter of the article. This study has received funding by Instituto de Salud Carlos III FEDER (PI09/01153 and PI11/01296). The statistics expert is one of the authors (MC). Institutional review board approval was obtained. The institutional review board of Parc de Salut Mar (CEIC-Parc de Salut MAR) determined that informed consent was not required for this study. Some study subjects or cohorts have been previously reported by Sala et al. (Implementation of digital mammography in a population-based breast cancer screening program: effect of screening on recall rate and cancer detection. Radiology, 252(1):31-39, 2009). Methodology: retrospective, cohort study/observational, performed at one institution.
}

Open Access This article is distributed under the terms of the Creative Commons Attribution Noncommercial License which permits any noncommercial use, distribution, and reproduction in any medium, provided the original author(s) and the source are credited.

\section{References}

1. Vinnicombe S, Pinto Pereira SM, McCormack VA, Shiel S, Perry N, Dos Santos Silva IM (2009) Full-field digital versus screen-film mammography: comparison within the UK breast screening program and systematic review of published data. Radiology 251:347-358

2. Nederend J, Duijm LE, Louwman MW, Groenewoud JH, Donkersvan Rossum AB, Voogd AC (2012) Impact of transition from analog screening mammography to digital screening mammography on screening outcome in the Netherlands: a population-based study. Ann Oncol 23:3098-3103

3. van Luijt PA, Fracheboud J, Heijnsdijk EAM, den Heeten GJ, de Koning HJ (2013) Nation-wide data on screening performance during the transition to digital mammography: observations in 6 million screens. Eur J Cancer 49:3517-3525

4. Domingo L, Romero A, Belvis F et al (2011) Differences in radiological patterns, tumour characteristics and diagnostic precision between digital mammography and screen-film mammography in four breast cancer screening programmes in Spain. Eur Radiol 21: 2020-2028

5. Bluekens AM, Holland R, Karssemeijer N, Broeders MJ, den Heeten GJ (2012) Comparison of digital screening mammography and screen-film mammography in the early detection of clinically relevant cancers: a multicenter study. Radiology 265:707-714

6. Hofvind S, Skaane P, Elmore JG, Sebuodegard S, Hoff SR, Lee CI (2014) Mammographic performance in a population-based screening program: before, during, and after the transition from screen-film to full-field digital mammography. Radiology 272:52-62

7. Sala M, Salas D, Belvis F et al (2011) Reduction in false-positive results after introduction of digital mammography: analysis from four population-based breast cancer screening programs in Spain. Radiology 258:388-395

8. Del Turco MR, Mantellini P, Ciatto S et al (2007) Full-field digital versus screen-film mammography: comparative accuracy in concurrent screening cohorts. AJR Am J Roentgenol 189:860-866

9. Hambly NM, McNicholas MM, Phelan N, Hargaden GC, O'Doherty A, Flanagan FL (2009) Comparison of digital mammography and screen-film mammography in breast cancer screening: a review in the Irish breast screening program. AJR Am J Roentgenol 193: $1010-1018$

10. Hoff SR, Abrahamsen AL, Samset JH, Vigeland E, Klepp O, Hofvind S (2012) Breast cancer: missed interval and screeningdetected cancer at full-field digital mammography and screen-film mammography-results from a retrospective review. Radiology 264 : 378-386

11. Nederend J, Duijm LEM, Louwman MWJ et al (2014) Impact of the transition from screen-film to digital screening mammography on interval cancer characteristics and treatment - a population based study from the Netherlands. Eur J Cancer 50:31-39

12. Vigeland E, Klaasen H, Klingen TA, Hofvind S, Skaane P (2008) Full-field digital mammography compared to screen film mammography in the prevalent round of a population-based screening programme: the Vestfold County Study. Eur Radiol 18:183-191

13. American College of Radiology (ACR) (2003) Breast Imaging Reporting and Data System Atlas (BI-RADS ${ }^{\circledR}$ Atlas). ACR, Reston

14. Perry N (2006) European guidelines for quality assurance in breast cancer screening and diagnosis, 4th edn. Office for Official Publications of the European Communities, Luxembourg 
15. Domingo L, Sala M, Servitja S et al (2010) Phenotypic characterization and risk factors for interval breast cancers in a population-based breast cancer screening program in Barcelona, Spain. Cancer Causes Control 21:1155-1164

16. Drukker CA, Schmidt MK, Rutgers EJ et al (2014) Mammographic screening detects low-risk tumor biology breast cancers. Breast Cancer Res Treat 144:103-111

17. Weigel S, Heindel W, Heidinger O, Berkemeyer S, Hense HW (2014) Digital mammography screening: association between detection rate and nuclear grade of ductal carcinoma in situ. Radiology 271:38-44

18. Allred DC (2010) Ductal carcinoma in situ: terminology, classification, and natural history. J Natl Cancer Inst Monogr 2010:134-138

19. Jones JL (2006) Overdiagnosis and overtreatment of breast cancer: progression of ductal carcinoma in situ: the pathological perspective. Breast Cancer Res 8:204
20. Sala M, Comas M, Macià F, Martinez J, Casamitjana M, Castells X (2009) Implementation of digital mammography in a populationbased breast cancer screening program: effect of screening round on recall rate and cancer detection. Radiology 252:31-39

21. Heddson B, Ronnow K, Olsson M, Miller D (2007) Digital versus screen-film mammography: a retrospective comparison in a population-based screening program. Eur J Radiol 64:419-425

22. Lewin JM, Hendrick RE, D'Orsi CJ et al (2001) Comparison of fullfield digital mammography with screen-film mammography for cancer detection: results of 4,945 paired examinations. Radiology 218:873-880

23. Comas M, Arrospide A, Mar J et al (2014) Budget impact analysis of switching to digital mammography in a population-based breast cancer screening program: a discrete event simulation model. PLoS One 9:e97459

24. Svendsen AL, Olsen AH, Euler-Chelpin M, Lynge E (2006) Breast cancer incidence after the introduction of mammography screening: what should be expected? Cancer 106:1883-1890 